\title{
The Striated Urogenital Sphincter Muscle in the Female
}

\author{
THOMAS M. OELRICH \\ Department of Anatomy, University of Michigan, Ann Arbor, Michigan 48109
}

\begin{abstract}
Striated muscle associated with the female urethra and vagina constitute a continuous mass which appropriately may be called the urogenital sphincter. Though continuous, the muscle may be separated into two parts-one that surrounds the urethra, and the other surrounding the urethra and vagina. The individual muscle fibers are small and are embedded in connective tissue and infiltrated with smooth muscle which obscures the visibility of the muscle to gross dissection. Developmentally the muscle primordium is laid down around the urogenital sinus and urethra early, and foreshadows the anatomical arrangement that is maintained in the adult with little change.

The urogenital sphincter muscle extends from the base of the bladder where it lies within the pelvic cavity and continues through the urogenital hiatus of the pelvic diaphragm to expand around the vagina in the perineum. Additional fibers attach to the ischiopubic rami and constitute a compressor of the urethra. As a result there is no superior fascia of the so-called "urogenital diaphragm" which closes off a deep perineal compartment or forms a floor of the urogenital hiatus.
\end{abstract}

Few anatomists and probably fewer students have seen the urogenital sphincter muscle in the female. Indeed the original literature and early reference texts of gross anatomy provide descriptions that are incomplete, ambiguous, and controversial. Only two muscles, $M$. sphincter urethrae, and $M$. transversus perinei profundus, are listed in the most recent Nomina Anatomica (Fourth edition).

Early anatomists recognized muscle fibers surrounding the urethra. Wilson (1809) described the male urethral sphincter and indicated that he had seen a similar muscle in the female surrounding the urethra and attaching to the pubis in front and the perineal body behind. Guthrie (1845), in describing the male urethral sphincter as lying between two layers of deep perineal fascia, indicated that the same muscle could be seen in the female, but with lateral attachments.

Luschka (1864) described an $M$. constrictor cunni profundus as a type of vaginal sphincter lying deep to the vestibular bulb and extending forward to the sides and ventral to the urethra. According to his description this muscle continued along the urethra to the base of the bladder. The fibers did not surround the urethra, but only attached to the anterior wall of the vagina. Luschka also described a $M$. transversus perinei profundus, which extended from the ischium to the perineal body behind the greater vestibular gland.
Henle (1873) unfortunately combined the muscles described by Luschka into a single unit, the $M$. transversus perinei profundus, and furthermore described the urogenital diaphragm as consisting of two aponeuroses with the $M$. transversus perinei profundus between these layers. He accurately indicated that the diaphragm contained quantities of smooth muscle in which the striated muscle fibers were recognizable only singly and sometimes not at all. He did not recognize the urethral sphincter muscle, but he stated that the muscle of the urogenital diaphragm could not be a urethral sphincter. Henle's description has been the source of much subsequent confusion, not only for giving the term $M$. transversus perinei profundus to all of the muscle of the region but by describing the so-called "urogenital diaphragm," which no subsequent researchers have confirmed yet which all subsequent textbook writers have reiterated with unerring accuracy.

Leeshaft (1884) described the urogenital muscle including a $M$. constrictor urethrae, which surrounded the urethra and extended from the base of the bladder to the perineal membrane, and a $M$. constrictor vestibulae $s$. sphincter vaginae (M. constrictor cunni profundus of Luschka), but indicated that it did not

Received January 21, 1982; accepted October 11, 1982. 
pass behind the vagina. While understanding the continuity of the two previous muscles he defined a $M$. transversus urethrae and a $M$. transversus vaginae extending from the lateral wall of the urethra and the anterior wall of the vagina to the descending process of the pubis. He also included a separate $M$. transversus perinei profundus extending from the ischial tuberosity to the perineal body behind the vagina. Leeshaft recognized that the fascia of the pelvis did not separate the pelvis from what was called the urogenital diaphragm by Henle, but that the fascias formed a urethrovaginal capsule continuous with the pelvic cavity above and closed off below by a perineal membrane.

Holl (1897) reiterated the descriptions of previous workers and included the $M$. transversus perinei profundus and the $M$. sphincter urethrae s. urethrovaginalis as parts of the urogenital diaphragm of Henle. Holl considered the $M$. transversus urethrae of Leeshaft to be part of the sphincter urethrae muscle, and the $M$. transversus vaginae of Leeshaft as part of the $M$. transuersus perinei profundus.

Kalischer (1900), in the most complete and accurate description, reviewed the findings of all previous researchers and added his own observations concluding that the muscle consisted of a $M$. sphincter urogenitalis with two parts-a pars urethrovaginalis and a pars urethralis. The pars urethrovaginalis enclosed the urethra and vagina, and the pars urethralis encircled the middle third of the urethra. Kalischer recognized a series of transverse fibers called the $M$. urethrotransversales "if they are at all present," which he indicated to be very weakly developed, but he observed that they were like the $M$. transversus urethrae and the $M$. transversus vaginae of Leeshaft, not the $M$. transversus perinei profundus of Henley and Holl. Kalischer recognized that the true $M$. sphincter urethrae lies within the urogenital hiatus of the pelvic diaphragm and extends to the bladder.

Recent workers have limited their observations to the urethra and its striated sphincter. Ricci et al. (1950) reiterated that there was a striated sphincter surrounding the middle third of the urethra while indicating that they found no fascial sheets "(superior diaphragmatis urogenitalis inferior and superior) and no compartment (superficial and deep perineal) pierced by the urethra."

Gil Vernet (1953) described and illustrated the urethral sphincter muscle in the middle third of the urethra and commented upon the more proximal fibers that reach the base of the bladder. He described the sphincter as consisting of completely circular fibers in his young specimens. He perceived that the more distal fibers of the sphincter of the urethra inserted into the vaginal wall.

Hutch and Rambo (1967) identified the urethral sphincter extending to the bladder neck and surrounding the middle third of the urethra, though they conventionally utilized the "urogenital diaphragm" as its location.

With few exceptions, modern textbooks of anatomy and reference texts give sketchy and essentially incorrect descriptions of the female urogenital musculature. Those authors who do refer to the literature seem to make an arbitrary selection of their reference descriptions. Thus, though some aspects of the musculature have been accurately described, confusion persists in anatomical accounts of this region. As a result, no complete and accurate illustrations are available. Without a doubt this confusion is due in part to the difficulty in dissecting the region since the muscle is embedded in dense investments of connective tissue; the muscle fibers are smaller than striated fibers throughout the rest of the body, and there are large quantities of smooth muscle associated with the striated fibers. Hayek (1960) compared the size of the individual striated fiber of the urethral sphincter muscle with other striated muscles of the human and found them to be $30 \%$ smaller. Oelrich (1980) described the size of the muscle fiber and connective tissue investment of the muscle fiber in the male, and Gosling (1979) defined these muscle fibers as exclusively "slow-twitch" in distinction to other mixed (slow- and fast-twitch) striated fibers found throughout the body.

It is the purpose of this paper to describe the striated urogenital sphincter musculature in the female, to adjudicate the various descriptions extant, and to relate these muscle masses to the various fascial planes and compartments of the pelvis and perineum.

\section{MATERIALS AND METHODS}

Gross dissections were attempted under low magnification, but these efforts were initially found to be useless since dense connective tissue and associated smooth muscle obscured the striated muscle fibers. Subsequently the urethra, vagina, rectum, and perineum were removed en bloc, sectioned serially, and stained with trichrome stains. Sections were made in transverse, sagittal, and frontal planes. Each half of an individual specimen was sectioned in a different plane. The pelves from 14 fetuses, 
ranging from $66 \mathrm{~mm} \mathrm{CR}$ to term were serially sectioned and examined in conjunction with 29 series of fetuses from the University of Michigan embryology collection. Fourteen pelves from term to 37 years were serially sectioned and examined. After the anatomy was confirmed by sectioned material the region was dissected under low magnification. These additional 29 gross dissections from term to 30 years proved to be productive and adequately confirmed spatial and fascial relationships.

\section{OBSERVATIONS \\ Early Development}

The disposition of the urogenital sphincters can be understood best by examining the developing fetus, which shows the arrangement of mesodermal condensations ultimately differentiating to striated muscle. In a $60-\mathrm{mm} C R$ fetus (Fig. 1), just below the vesical neck, the mesenchyme thickens anterior to the vesicourethral canal. Dorsal to this canal are the fused paramesonephric ducts which join the urogenital sinus at the level of the tuberculum sinuale (Fig. 2) (Müllerian tubercle) and thus separate off the primitive urethra above, from the urogenital sinus below. The thickened mesenchyme lies ventral and lateral to this junction (Fig. 2). Caudaliy the thickened mesenchyme completely surrounds the urogenital sinus (Fig. 3). The proximal fibers will become a urethral sphincter and the distal fibers a urethrovaginal sphincter.

In an 111-mm CR fetus (Fig. 4) sectioned in a frontal plane, the thickening mesenchyme extends from the base of the bladder to the vestibular bulb demonstrating the continuity of the two parts. With the location of the pelvic diaphragm (Fig. 4), it is evident that the urogenital sphincter primordium lies in the pelvic cavity, in the urogenital hiatus (between the halves of the pelvic diaphragm) and in the perineum.

In a 140-mm CR fetus (Fig. 5) on a sagittal plane just lateral to the urethral wall, the entire urogenital sphincter primordium can be seen extending from the base of the bladder to the vestibular bulb. Fibers can be identified between the primitive urethra and the paramesonephric duct and farther caudally fibers surrounding the beginning of the urogenital sinus. Immediately caudal to the primordial sphincter are the vestibular bulb and the greater vestibular gland, which maintains these positions in the adult. In the same series on the midsagittal plane (Fig. 6), the relations between the tuberculum sinuale and the lower border of the sphincter can be established. Fibers of the primordial sphincter, behind the tuberculum sinuale, do not intermingle with the external anal sphincter but lie at a higher level.

In a slightly older fetus, $163 \mathrm{~mm}$ CR, (Fig. 7), sphincter fibers can be identified on the midline between the primordial urethra and the primordial vagina, thus establishing the complete primordial urethral sphincter muscle.

In the 240-mm CR fetus the mesenchyme has completely differentiated so that striated fibers are evident. Simply described, the primordial urogenital sphincter in the female consists of two distinct elements: 1) a sphincter of the urethra, and 2) a sphincter of the urogenital sinus. The elements are continuous and extend from the base of the bladder to the perineal membrane lying within the pelvic cavity, in the urogenital hiatus, and in the perineum. Though modifications of this arrangement occur throughout subsequent growth, the basic pattern does not change. This distinguishes the development of the male from the female, as in the male the development of the prostate shapes and displaces the urethral sphincter (Oelrich, 1980).

Growth to term shows only a single modification in which the lower border of the urogenital sphincter extends laterally to the ischiopubic ramus below the pelvic diaphragm. This is not readily definable early because of the disproportionately large size of the developing vagina which fills the space between the ischiopubic rami.

In the first two decades gross dissection of the sphincter mechanism is relatively productive with low magnification. However, the subsequent increases in the density of connective tissue and smooth muscle obstruct the visibility of the striated muscle so that dissection becomes more difficult later.

\section{Adult}

Complete development of the urogenital sphincters changes the proportional distribution of the muscle seen in earlier decades. Kalischer (1900) emphasized the continuity of the muscle masses by naming them the $M$. sphincter urogenitalis with a pars urethralis and a pars urethrovaginalis. The use of such terminology seems logical and will be maintained here. In the adult these two parts lie in distinctly different anatomical areas though they are continuous with one another. The pars urethralis lies within the pelvic cavity; the pars urethrovaginalis lies in the perineum. The per- 


\section{Abbreviations}

\begin{tabular}{ll} 
AL & Arcuate pubic ligament \\
AS & External anal sphincter \\
B & Bladder \\
BS & Bulbospongiosus muscle \\
CCC & Corpus cavernosum clitoris \\
CU & Compressor urethrae muscle \\
IC & Ischiocavernosus muscle \\
IP & Ischiopubic ramus \\
IR & Ischial ramus \\
MD & Fused paramesonephric ducts \\
OI & Obturator internus muscle \\
PD & Pelvic diaphragm \\
PF & Pelvic fascia \\
PM & Perineal membrane \\
PS & Pubic symphysis \\
R & Rectum \\
SM & Smooth muscle of urethrovaginal compartment \\
SU & Urogenital sinus \\
TS & Tuberculum sinuale (Müllerian tubercle) \\
TV & Transverse vaginal muscle \\
U & Urethra \\
UGS & Urogenital sphincter primordium \\
US & Urethral sphincter \\
UVS & Urethrovaginal sphincter \\
V & Vagina \\
VB & Vestibular bulb \\
VG & Greater vestibular gland \\
VU & Vesicourethral canal \\
VW & Vaginal wall \\
\hline
\end{tabular}

ineal part (Fig, 15) parallels the ischiopubic rami and is therefore at an angle of approximately $130^{\circ}$ with the urethral part (Fig. 17). It should be remembered that the urethra itself is not a straight tube but is concave ventrally. The continuity between the two parts lies in the urogenital hiatus of the pelvic diaphragm just dorsal to the pubic symphysis.

\section{Sphincter Urogenitalis (Pars Urethrae)}

$M$. sphincter urethrae PNA. The $M$. sphincter urethrae surrounds the urethra in the middle third of its length for approximately $1.5 \mathrm{~cm}$ (Figs. 13, 16, 17). It begins at the base of the bladder, and caudally is continuous with the perineal component of the urogenital sphincter, $M$. compressor urethrae. As previously expressed in the developmental considerations, the undifferentiated mesenchyme completely surrounds the urethra; however, in the completely developed sphincter, the striated fibers vary in their density and degree of encirclement of the urethra. In the child striated fibers completely surround the urethra (Fig. 8). In the adult, the sphincter is thickest on its ven- tral side and thins as it passes to the dorsal side of the urethra (Fig. 11). There appears to be a dorsal septum or a raphe into which the fibers attach (Fig. 11). The size of this septum and the degree to which some striated fibers cross the midline vary with the individual. The presence of a fibrous septum or raphe would change the manner in which the sphincter fibers constrict the urethra, although the major content of this apparent septum is the circular layer of the smooth muscle of the urethra. The orientation of the urethral sphincter fibers is predominantly circular (Figs. 11, 13, 17), though groups of fibers run obliquely within the sphincter. In the proximal part some fibers extend vertically into the bladder muscle. A few peripheral fibers, instead of encircling the urethra, attach to the vaginal wall or into adjacent connective tissue (the endopelvic fascia). There is an infiltration of smooth muscle throughout the length of the sphincter. The striated fibers appear to lie in the plane of the circular smooth muscle of the urethra. Smooth muscle bundles from the bladder overlie the proximal end of the urethral sphincter (Fig. 13).

The location of the urethral sphincter corresponds precisely with the area of highest ure-

Fig. 1. Cross section of a female urethra in a 60-mm CR fetus just caudal to the bladder. $\times 17$.

Fig. 2. Cross section of a female urethra in a $60-\mathrm{mm}$ CR fetus at the level of the tuberculum sinuale. $\times 17$.

Fig. 3. Cross section of a female urethra in a $60-\mathrm{mm}$ CR fetus through the urogenital sinus. Note the position of the pelvic diaphragm. $\times 17$.

Fig. 4. Coronal section of a 111-mm CR female fetus through the bladder, urethra, and urogenital sinus. $\times 10$.

Fig. 5. Saggital section of a $140-\mathrm{mm}$ CR female fetus demonstrating the extent of the developing urogenital sphincter primordium. $\times 9$.

Fig. 6. Median section of a 140-mm CR fetus. Note portion of urogenital sphincter primordium between tuberculum sinuale and rectal wall. $\times 9$.

Fig. 7. Median section of a 163-mm CR female fetus. Note urethral sphincter primordium between urethra and primordial vagina. $\times 9$. 


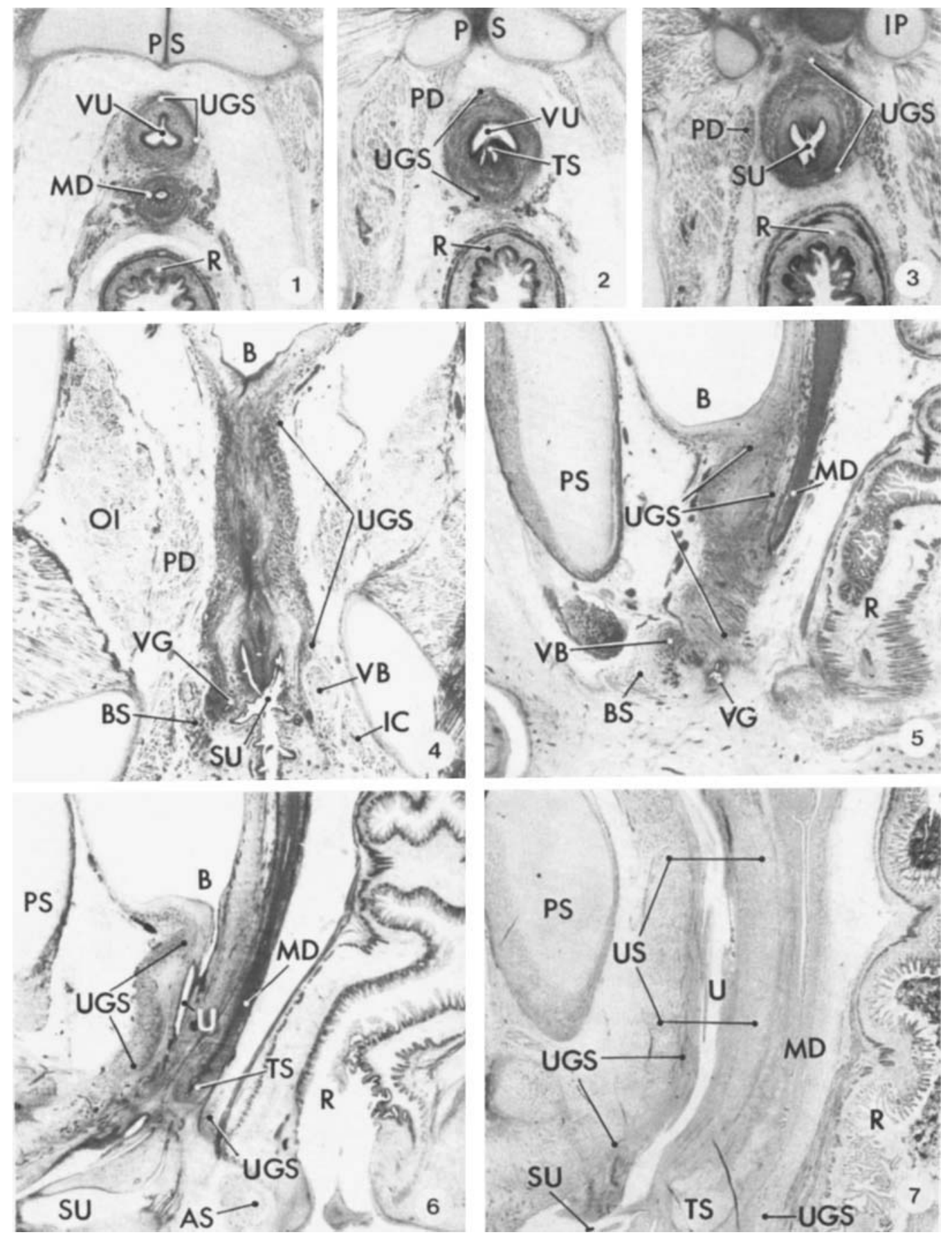



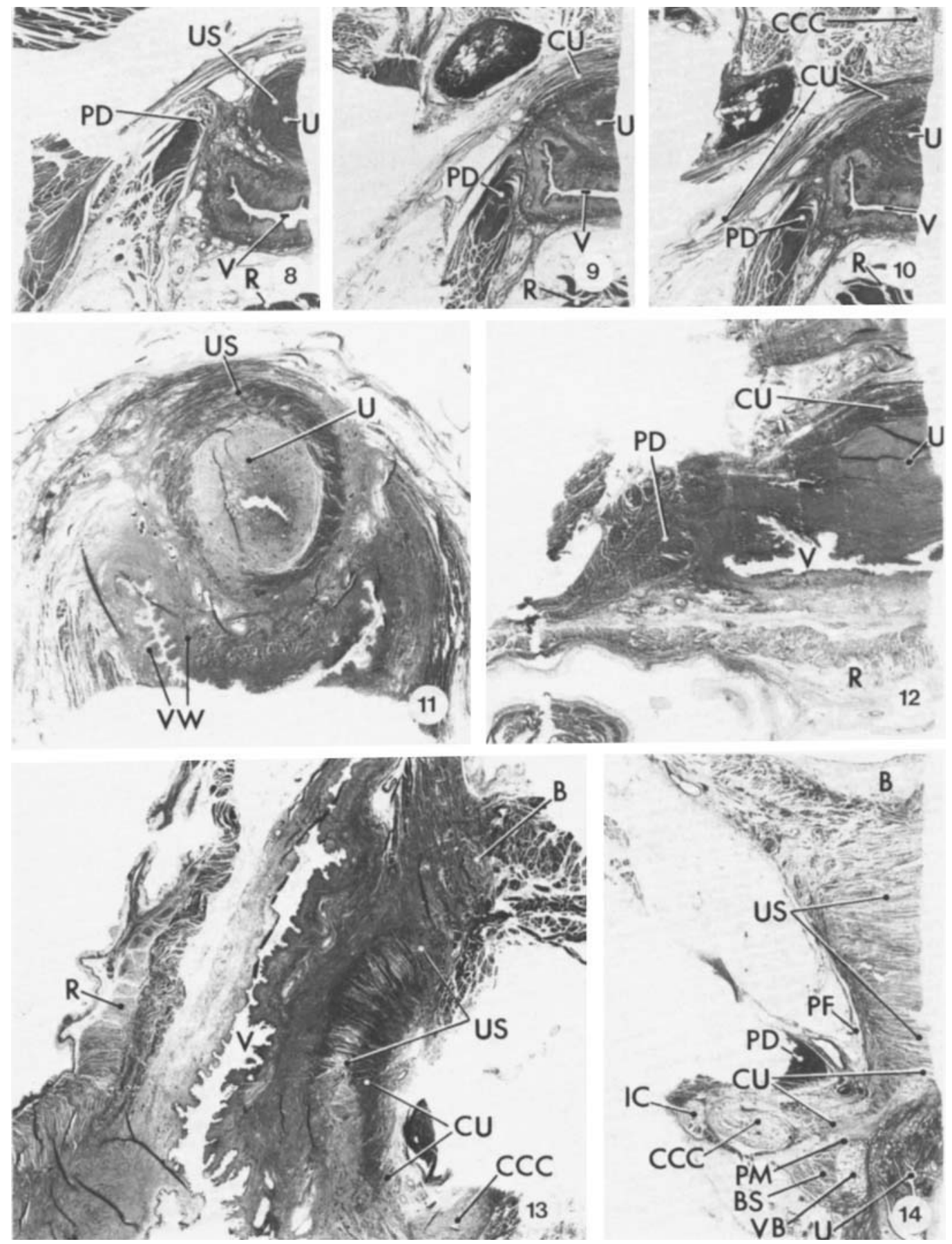
thral closure pressure as measured by Tanagho and Smith (1968).

\section{Sphincter Urogenitalis (Pars \\ Urethrovaginalis)}

$M$. compressor urethrae, nom. nov. (M. transversus urethrae). Distal to and directly continuous with the lower border of the urethral sphincter (Figs. 9, 10, 12-17) is a bundle of fibers which had been identified previously, but was described in a variety of different ways. The muscle begins as a small tendon approximately $2 \mathrm{~mm}$ in diameter attaching to the ischiopubic ramus laterally at a point near the anterior border of the ischial tuberosity or on a plane at the posterior border of the vagina (Figs. 16, 17). The muscle extends forward toward the anterior surface of the urethra, thickens to a band of approximately $6 \mathrm{~mm}$ wide (Figs. 9, 12, 13), and then becomes continuous

Fig. 8. Cross section of the left half of a female pelvis 1 year old, through the urethral sphincter muscle just caudal to the bladder. Note position of the pelvic diaphragm. $\times 2$.

Fig. 9. Cross section of the left half of a female pelvis 1 year old demonstrating the upper border of the compressor urethrae muscle anterior to the urethra. $\times 2$.

Fig. 10. Cross section of the left half of a female pelvis 1 year old demonstrating the lateral extent of the compressor urethrae muscle. Note absence of fibers between the urethra and vagina; also note the proximity of the corpus cavernosum clitoris to the anterior border of the compressor urethrae muscle. $\times 2$.

Fig. 11. Crose section of an adult urethra and vagina demonstrating the urethral sphincter muscle at the level of the urogenital hiatus of the pelvic diaphragm. Note apparent septum in the posterior portion of the sphincter. Age 33 years; $\times 1.8$.

Fig. 12. Cross section of an adult urethra and vagina demonstrating the upper edge of the compressor urethrae muscle. Compare with Figures 9 and 10 . Age 29 years; $x$ 1.8.

Fig. 13. Sagittal section of an adult female urethra and vagina. The section is slightly off of the midline so that the entire urethral sphincter muscle can be viaualized. Note the continuity between the urethral sphincter muscle and the compressor urethrae muscle. Age 29 years; $\times 1.8$.

Fig. 14. Coronal section of a female pelvis demonstrating continuity of the urethral sphincter muscle and the compressor urethrae. Note absence of any fascia which may separate the pelvic structures from the perineal structures (the so-called superior fascia of the urogenital diaphragm). Age 16 years; $\times 2.6$. with the corresponding fibers of the opposite side of the body, forming a broad arcing muscle (Figs. 10, 15). As the band reaches the urethra it rotates, so that its dorsal edge becomes inferior (Fig. 15) (located at the plane of the arcuate pubic ligament) and its ventral edge becomes superior. The superior edge lies within the urogenital hiatus of the pelvic diaphragm and is continuous with the lower fibers of the ventral part of the urethral sphincter (Figs. 13, $14,16,17$ ) where it lies behind and deep to the pubic symphysis. Although most of the muscle passes ventral to the urethra (Figs. 12,13) some few deep fibers attach to the lateral wall of the urethra. Leeshaft (1884) named these latter fibers the $M$. transversus urethrae, though he was apparently not aware of the large mass of muscle passing ventral to the urethra. The $M$. compressor urethrae is continuous with the $M$. sphincter urethrovaginalis along the inferior border of the $M$. compressor (Figs. 16, 17). Functionally, the $M$. compressor urethrae can compress the urethra from its ventral position. Because it approaches the urethra parallel to the ischiopubic rami (at an angle of $130^{\circ}$ to the urethra), it additionally can have the effect of pulling the urethral meatus caudally and inferiorly (Figs. 15, 16). In combination with the action of the pelvic diaphragm (elevating the bladder), it assists in elongation of the urethra. Lapides (1958) emphasized the importance of urethral elongation as a means of providing continence.

Variations of this pattern do exist. Some fibers may appear to attach to the transverse ligament of the perineum, thus giving rise to an older term, $M$. ischiopubicus. Occasionally the $M$. compressor urethrae may join the urethrovaginal sphincter near the anterior border of the vagina rather than ventral to the urethra. Its size may be reciprocal with that of the $M$. sphincter urethrovaginalis.

M. Sphincter Urethrovaginalis (M. Sphincter Vaginae BNA, M. Sphincter Vestibuli, M. Constrictor Cunni Profundus). The M. sphincter urethrovaginalis (Figs. 15-17) is a thin, flat muscle approximately $5 \mathrm{~mm}$ broad which blends ventrally with the $M$. compressor urethrae. From the ventral side of the urethra its fibers extend dorsally along the lateral wall of the urethra and vagina immediately deep to the cephalic edge of the vestibular bulb. It is separated from the vestibular bulb by a barely perceptible, thin layer of the perineal membrane. None of its fibers pass between the urethra and vagina, 

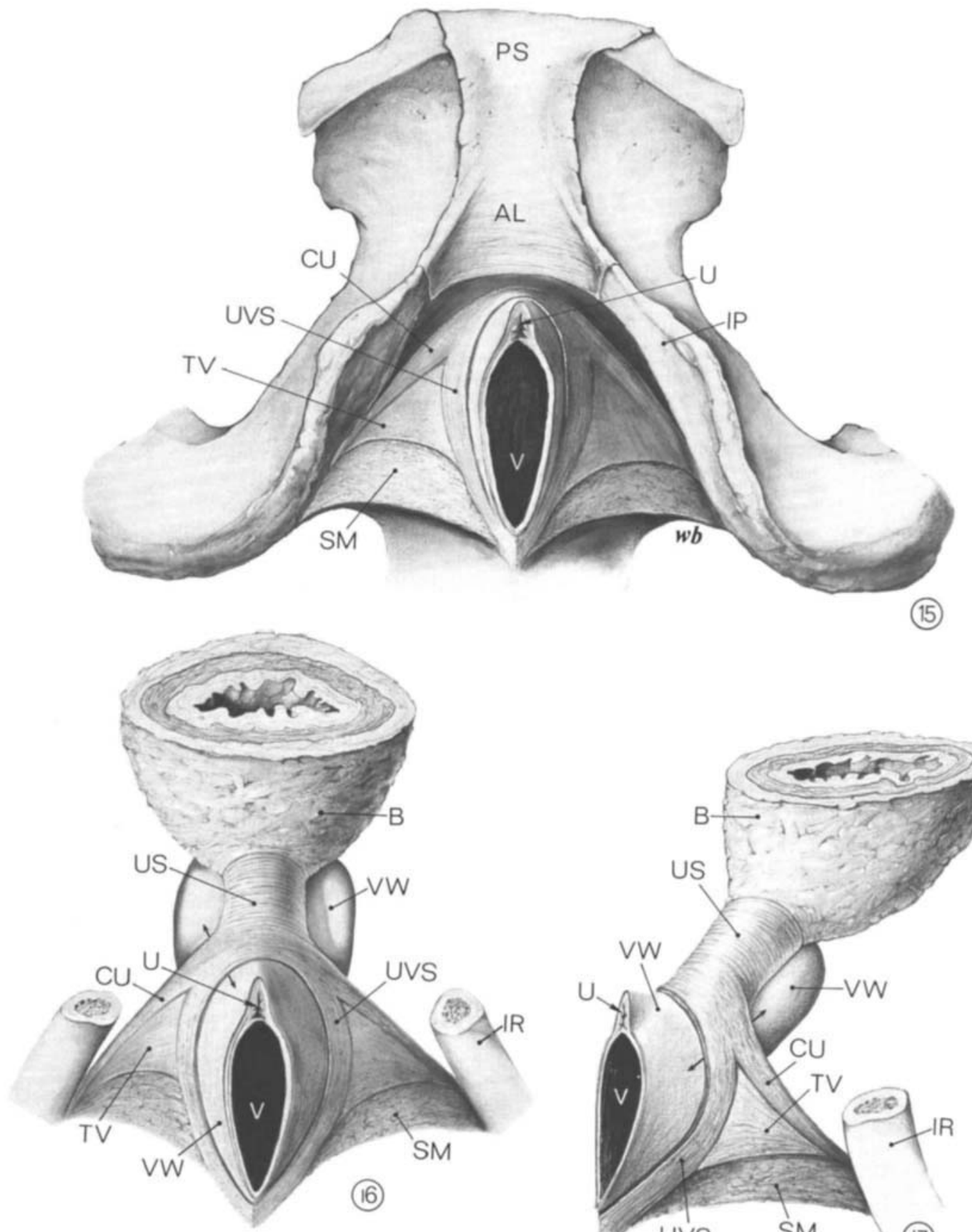

(15)

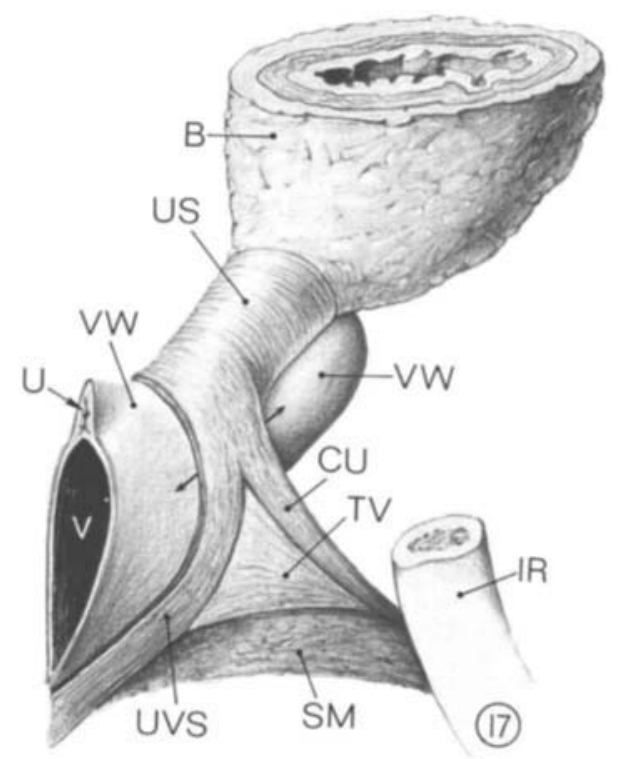

Fig. 15. Perineal view of the urogenital sphincter mugculature with the perineal membrane removed. Age 27 years; $\times 0.6$.

Fig. 16. View of complete urogenital sphincter musculature, bladder, and vagina with the pubic symphysis removed and the ischial rami spread. Arrow indicates continuity of the vaginal wall beneath the muscle. Age 27 years; $\times 0.6$.
Fig. 17. Oblique view of complete urogenital sphincter muscles, bladder, and vagina. Arrow indicates continuity of the vaginal wall beneath the muscle. Age 27 years; $\times 0.6$. 
nor do any of its fibers pass in front of or ventral to the vagina. Fibers continue dorsally to pass behind the vagina and interdigitate with the corresponding muscle of the opposite side. In the first two decades the muscle is distinct and proportionately small. With the increased density of the connective tissue of the perineal body, it becomes difficult to identify fibers behind the vagina.

The term $M$. sphincter vaginae is historically appropriate; however, the muscle does not exclusively surround the vagina, and is morphologically and functionally a urethrovaginal sphincter. In fact, the two muscles $M$. compressor urethrae and $M$. sphincter urethrovaginalis share the mass of muscle lying ventral to the urethra but caudal to the $M$. sphincter urethrae (Figs. 12, 13, 16, 17). The two muscles may be reciprocal in size. The continuity of this muscle with the $M$. compressor urethrae would imply that they complement one another in compression, retraction, and elongation of the urethra.

M. Transversus Vaginae. In additional layer of striated muscle $(M$. transversus vaginae of Leeshaft, 1884) parallels the $M$. compressor urethrae and radiates from it to attach to the anterior portion of the lateral wall of the vagina, attaching just above the urethrovaginal sphincter (Figs. 15-17). The M. compressor urethrae and $M$. transversus vaginae form a continuous muscle, but, because of the different course, thickness, and attachments, it seems appropriate to maintain individual names for the various parts. Though its size and extent do vary, these fibers form a very thin, fan-shaped muscle that seldom attaches to the posterior half of the vagina.

The $M$. transversus perineus profundus was originally described by Luschka (1864) as extending from the ischial tuberosity to the perineal body. Subsequently, because others may not have been able to find the muscle (Henley, 1873; Holl, 1897), it became synonymous with the $M$. transversus vaginae and ultimately with the $M$. transversus urethrae. Kalischer could not identify the muscle, and neither can this author. It is entirely possible that misidentifications have occurred because of the variable extent of the perineal membrane. In many specimens this membrane appears to extend to the midpoint of the rectum. In this manner fibers of the $M$. transversus perineus superficialis may easily be contained within this fascia. The posterior border of the perineal membrane can usually be identified by the presence of arteries, veins, and nerves passing through it.

Urethrovaginal Compartment. The urogenital sphincter muscle lies above or cephalic to the perineal membrane (Fig. 14) which separates these muscles from the ischiocavernosis and the bulbospongiosus muscles and the associated cavernous bodies. While surrounding the urethra and vagina the muscle is enclosed within a compartment formed by the perineal membrane inferiorly and a superior fascia, continuous through the urogenital hiatus of the pelvic diaphragm with the fascias of the pelvis. This is not the so-called "urogenital diaphragm" described by Henle, which he indicated closed off the urogenital hiatus, but a urethrovaginal capsule (compartment) continuous with the pelvic cavity as described by Leeshaft.

The urogenital hiatus in the female is much reduced in comparison to the male since fibers of the pubococcygeus muscle of the pelvic diaphragm insert into the lateral wall of the vagina. The hiatus is therefore anterior to the vagina, and transmits only the urethra and its muscle (Fig. 14).

The combined superior fascia of the pelvic diaphragm (transversalis) and the attached endopelvic fascia pass around the border of the urogenital hiatus (Fig. 14). Continuing through the hiatus, this layer passes dorsally to join the posterior border of the perineal membrane and form an "apparent" diaphragm. Laterally this fascia joins the obturator fascia (or fascia lunata) extending to the ischiopubic ramus. Anteriorly the thickened endopelvic fascia of the bladder and urethra passes through the urogenital hiatus joining the anterior edge of the perineal membrane as the transverse ligament of the perineum. These fascias collectively form a compartment which may appear as a diaphragm in the perineum while superiorly it is open and directly continuous with the pelvic cavity (Fig. 14). If one considers the specializations of the endopelvic fascia, the medial and lateral pubovesical ligaments, one might say that the compartment is limited superiorly by these.

This compartment, in addition to the urogenital sphincter muscle, contains an extensive mass of smooth muscle. It is difficult to separate the smooth muscle from the layers of fascia exiting from the pelvis. The smooth muscle extends throughout the perineal region from the ischiopubic rami to the lateral wall of the urethra and vagina lying between the perineal 
membrane and the superior fascia of the urethrovaginal compartment (Figs. 15-17). The definitive character of the posterior border of the perineal membrane is due in part to the presence of this smooth muscle mass. In the first decade and a half there is only slight evidence of smooth muscle. The quantity seems to increase with age, and the striated component becomes less and less evident. The fact that the striated muscle is not well fasciculated and is covered with a relatively thick muscular fascia makes the separation of the smooth muscle from the striated muscle most difficult.

As mentioned previously, variations do occur with the characteristics and proportional relations of the striated muscles in the first decades. Increase in the presence of smooth muscle and the development of the perineal body also obstruct structural interpretations. Within the multipara, as might be expected, the perineal component of the urogenital sphincter muscles is extremely difficult to identify.

\section{SUMMARY}

The striated urogenital sphincter muscle of the female consists of 1) a sphincter surrounding the middle third of the urethra, 2) a urethrovaginal sphincter surrounding the urethra and vaginal vestibule collectively, and 3) a compressor of the urethra arcing across the ventral side of the urethra. Other variable striated fibers may occur. These designated muscles are in continuity with one another ventral to the urethra, and are located within the pelvic cavity, in the urogenital hiatus of the pelvic diaphragm, and in the perineum. Smooth muscle fibers may interlace the striated fibers to a variable degree. The perineal components of the urogenital sphincter are contained in a urethrovaginal compartment that is continuous with the pelvic cavity.

\section{ACKNOWLEDGMENTS}

I wish to express appreciation for the art work provided by William Brudon.

This work was supported in part by a grant from the United States Public Health Service-NIH 5R01 GM 09773-02.

\section{LITERATURE CITED}

Gil Vernet, S. (1953) Patologia Urogenital. Tomo II, Vol. I. Biología y Patología de la Prostata. Edit. Paz Montalvo, Madrid.

Gosling, J. (1979) The structure of the bladder and urethra in relation to function. Urol. Clin. North Am., 6:31-38.

Guthrie, B.J. (1845) On the Anatomy and Diseases of the Urinary and Sexual Organs. Lea and Blanchard, Philadelphia.

Hayek, H. (1960) Das Faserkaliber in den Mm. transversus perinei und Sphincter urethrae. Zeitsch. Anat. Entwicklungggeschichte., 121:455-458.

Henle, J. (1873) Handbuch der Systematischen Anatomie des Menschen. Bd. II., Druck und Verlag von Friedrich Vieweg und Sohn, Braunschweig.

Holl, M. (1897) Die Muskeln und Fascien des Beckenausganges. In Handbuch der Anatomie des Menschen. Bardeleben., Gustav Fischer, Jena.

Hutch, J.A., and O.N. Rambo (1967) A new theory of the anatomy of the internal urinary sphincter and the physiology of micturation. J. Urol., 97:696-704.

Kalischer, O. (1900) Die Urogenitalmuskulatur des Dammes. Verlag von S. Karger, Berlin.

Lapides, J. (1958) Structure and function of the internal vesical sphincter. J. Urol, 80:341-353.

Leeshaft, P. (1884) Uber die Muskeln und Fascien der Dammgegend beim Weibe. Gegenbaurs Morphologisches Jahrbuch, 9:475-532.

Luschka, H. (1864) Die Anatomie des Menschlichen Beckens. Laupp and Siebeck, Tubingen.

Oelrich, T.M. (1980) The urethral sphincter muscle in the male. Am. J. Anat., 158:229-264.

Ricci, J.V., J.R. Lisa, and C.H. Thom (1950) The female urethra. Am. J. Surg., 79:499-505.

Tanagho, E., and D. Smith (1968) Mechanism of urinary continence. 1. Embryologic, anatomic and pathologic considerations. J. Urol., 100:640-646.

Wilson, J. (1809) A description of two muscles surrounding the membranous part of the urethra. Med. Chirurg. Soc. Lond. $1: 175-180$. 